\title{
ORIGINAL ARTICLE \\ Relationship quality and perceived social support in persons with spinal cord injury
}

\author{
F Tramonti ${ }^{1}$, A Gerini ${ }^{2}$ and G Stampacchia ${ }^{2}$
}

Study design: This is a cross-sectional study.

Objectives: The objective of this study was to examine the associations among the quality of couple relationship, perceived social support and health-related quality of life (HRQLL) in persons with spinal cord injury (SCl).

Setting: The study was conducted in Italy.

Methods: Forty-three persons with $\mathrm{SCl}$ were administered questionnaires for the evaluation of relationship quality (Dyadic Adjustment Scale), perceived social support (Multidimensional Scale of Perceived Social Support) and HRQoL (Short Form 36).

Results: Many significant correlations between the scores of relationship quality and social support were detected. Relationship quality also correlated with relevant measures of HRQoL, such as Mental Health and Vitality. No correlation between spousal support and HRQoL was found, whereas friends' support correlated with Physical Role Functioning.

Conclusions: Data suggest that the perception of social support is strictly related to relationship quality and that marital satisfaction might be related to relevant aspects of HRQoL more likely than social support itself.

Spinal Cord (2015) 53, 120-124; doi:10.1038/sc.2014.229; published online 16 December 2014

\section{INTRODUCTION}

Social support is a key topic in the spinal cord injury (SCI) literature, owing to the influence it can exert on adjustment and mental health. ${ }^{1-3}$ Nevertheless, the relationship between social support and health or psychological well-being is extremely complex, and the mere presence of social networks does not ensure that an actual and effective support is perceived by the recipient. Furthermore, in conditions such as SCI, in which physical limitations and medical complications can be paralleled by the perception of reduced autonomy, research studies are progressively shifting their focus to the relationship between social support and social skills. ${ }^{4}$ Indeed, as also emphasised in studies devoted to other medical conditions, social support can be a 'double-edged sword', as supportive behaviours are not always positive or functional and they can reinforce the perception of reduced autonomy. ${ }^{5}$ In addition, the same helping and protective behaviour can be interpreted in terms of empathy or as an attempt at control. ${ }^{6}$ Given these premises, in the present study, we aimed at examining the association between social support and relationship quality in persons with SCI, also considering the correlations of such variables with health-related quality of life (HRQoL).

Marital satisfaction has been identified as a key factor in shaping health and well-being, as positive intimate relationships are important predictors of quality of life (QoL) during adulthood, and they can influence physiological processes, as well as treatment adherence and health habits. ${ }^{7}$ On the contrary, the protective role of marriage and couple relationships vanish when their quality is not satisfactory, and negative relationships can become a source of 'social strain'. 8,9
This means that the presence of a couple relationship is often an important predictor of better adjustment and higher QoL, but dysfunctional relationships can be additional sources of distress instead of being helping resources. Similar to social support, also intimate relationships could be seen as double-edged swords. In this vein, some authors suggest to pay special attention to relationship quality when examining social support, and to distinguish between general and relationship-based social support. ${ }^{10}$

According to the available literature, there is evidence that marriage can be a strong predictor, in the long run, of life satisfaction in persons ageing with SCI. ${ }^{11}$ However, despite variable percentages across different countries, people with SCI are often single and their divorce rates are higher than those of the general population. ${ }^{12,13}$ Furthermore, studies in SCI populations confirm that the quality of social support is much more important than the quantity of support, and that the perception of not being passive recipients of support but people taking advantage of help for active social integration and participation is a crucial factor. ${ }^{4,13}$ For these reasons, further investigation is needed to deepen our knowledge of the associations between social support and relationship quality in SCI populations, especially for those relationships that are more relevant in terms of life cycle issues and impact on QoL.

To date, social support has been more likely to be related to marital status than to marital satisfaction, and few studies have been conducted on the satisfaction from couple relationships in SCI populations. ${ }^{14-17}$ Such studies have found that couple satisfaction is not necessarily compromised in persons with SCI. Therefore, even if

${ }^{1}$ Clinical Psychology Unit, Department of Neurosciences, Azienda Ospedaliero-Universitaria Pisana, Pisa, Italy and ${ }^{2}$ Center for Spinal Cord Injuries, Department of Neurosciences, Azienda Ospedaliero-Universitaria Pisana, Pisa, Italy

Correspondence: Dr F Tramonti, Clinical Psychology Unit, Department of Neurosciences, Azienda Ospedaliero-Universitaria Pisana, Via Sant'Andrea 7 (home address), 56127 Pisa, Italy.

E-mail: tramonti.francesco@gmail.com

Received 4 July 2014; revised 12 November 2014; accepted 14 November 2014; published online 16 December 2014 
traumatic events such as SCI can disrupt intimate relationships, those couples who positively react to such events could be no different, in terms of satisfaction, from couples of the so-called able-bodied people. As previously mentioned, our study aimed at examining the associations between relationship quality and perceived social support in persons with SCI, taking into account the relationships between such variables and HRQoL. We hypothesise that relationship quality is not only a variable significantly related to relevant aspects of QoL but also a key factor in the perception of actual social support. Evidence from studies devoted to other conditions seems to support such a hypothesis, ${ }^{18}$ and specific aspects of SCI make such an investigation worthwhile. Indeed, chronic disease conditions characterised by physical limitations can threaten couple relationships, as they can have a negative impact on intimacy and they can foster patterns of distancing or, at the opposite, symbiotic relationships rigidly characterised by the roles of patient and caregiver. ${ }^{19}$

\section{MATERIALS AND METHODS}

A sample of 43 persons with SCI was recruited. Such a sample was derived from the evaluation of 67 consecutive persons attending the Centre for spinal cord injuries of the University hospital of Pisa from January 2012 to October 2013. All the included persons were married or involved in common-law partnerships for at least 1 year, and only three persons started the relationship after the injury. Individuals who were injured within a time period of 6 months before our testing were excluded. None of the persons had concurrent brain injuries, which was considered an additional exclusion criterion owing to the possible impact on cognitive functioning.

\section{Table 1 Study population characteristics}

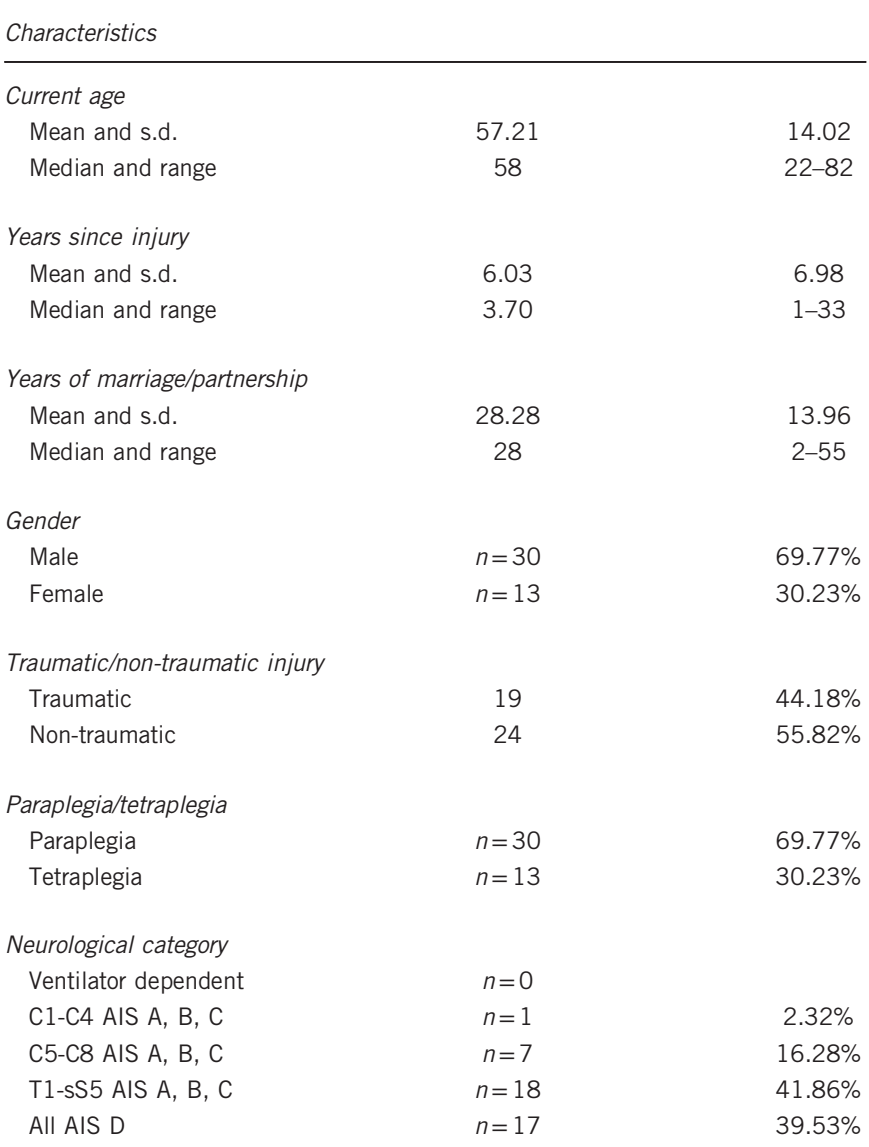

Abbreviation: AIS, American Spinal Injury Association Impairment Scale.

Descriptive statistics and neurological categories according to DeVivo et al. ${ }^{29}$
The quality of couple relationship was evaluated with the Dyadic Adjustment Scale (DAS), a questionnaire that provides a total score, as well as separate scores for its four subscales: Dyadic Cohesion, Dyadic Consensus, Affective Expression and Dyadic Satisfaction. ${ }^{20}$ The domains investigated by the questionnaire range from affective and sexual satisfaction to couple cohesion and shared values or activities. Thus, in this contest, the term dyadic adjustment does not refer to the process of coping with specific stressful events, but it is rather a measure of relationship quality.

Social support was measured by the use of the Multidimensional Scale of Perceived Social Support (MSPSS), a questionnaire that evaluates the perceived help from three main sources: a special person, the family and friends. ${ }^{21}$ All the individuals were asked to explicitly identify the special person, and only those who chose their partners were included in the study. The Short Form 36 was used for the evaluation of HRQoL. ${ }^{22}$ It is a well-known and widely used instrument that is based on eight subscales: Physical Functioning, Physical Role Functioning, Bodily Pain, General Health Perceptions, Vitality, Social Functioning, Emotional Role Functioning and Mental Health.

All the persons were also administered the Spinal Cord Independence Measure, a condition-specific scale that evaluates the degree of autonomy and physical functioning in activities of daily living such as washing, dressing and covering different distances. ${ }^{23}$ All the applicable institutional and governmental regulations concerning the ethical use of human beings were followed during the course of this research. Bivariate correlations (Pearson's $r$ ) between all the scales or subscales were calculated, and the differences between men and women were evaluated by the use of the $T$-test. A repeated measures analysis of variance was performed in order to estimate the within-group differences on the MSPSS subscale scores. Significance for $P<0.05$ was considered.

\section{RESULTS}

As it can be seen in Table 1, most of the individuals belong to the neurological categories of 'T1-sS5 American Spinal Injury Association Impairment Scale (AIS) A, B, C' or 'AIS D'. This means that most of them are persons with paraplegia or incomplete tetraplegia, and they may have acceptable levels of autonomy as also suggested by the average scores of the Spinal Cord Independence Measure (Table 2). The average total score of the DAS was very close to local norms. ${ }^{24}$ With respect to the MSPSS subscale scores, the repeated measures analysis of variance revealed that friends' support was significantly lower $\left(\mathrm{F}_{(2,41)}=15.98\right.$; $P=0.00)$ than both spousal and family support. No significant difference emerged between men and women in couple satisfaction or perceived social support, but the small sample size of the subgroups suggests caution in the interpretation of this result. Social support received from friends negatively correlated $(r=-0.37 ; \quad P=0.01)$ with age, whereas no correlation between age and spousal or family support was found. No correlation between the Spinal Cord Independence Measure scores and relationship quality or social support emerged.

Many significant correlations between the DAS and MSPSS scores were found, suggesting a strong interdependence between relationship quality and social support (Table 3). Measures of relationship quality, such as the DAS total scores and Affective Expression, not only correlated with spousal support but also with family support. Further correlations were also found between relationship quality and HRQoL (Table 4). In more detail, Affective Expression and the total scores of the DAS scale correlated with Vitality and Mental Health. The latter also correlated with Dyadic Consensus and Dyadic Satisfaction. On the contrary, the only correlation found between HRQoL and social support was that concerning Physical Role Functioning and friends' support $(r=0.36 ; P=0.02)$.

\section{DISCUSSION}

The data from this study confirm that marital satisfaction of persons with SCI can be as good as that of the general population, and that marital 
Table 2 Mean scores with s.d. of all the scales and subscales

\begin{tabular}{|c|c|c|c|c|c|c|c|c|c|}
\hline Mean & 17.91 & 37.32 & 9.05 & 50.77 & 115.05 & 24.35 & 24.46 & 19.51 & 68.32 \\
\hline \multirow[t]{2}{*}{ s.d. } & 12.04 & 10.86 & 2.57 & 12.08 & 22.42 & 4.64 & 5.66 & 5.48 & 11.05 \\
\hline & $P F$ & PRF & $B P$ & GHP & $V T$ & $S F$ & $E R F$ & $M H$ & SCIM \\
\hline Mean & 18.25 & 21.51 & 51.77 & 54.32 & 57.44 & 57.00 & 57.94 & 62.77 & 57.05 \\
\hline
\end{tabular}

Abbreviations: Aff Exp, Affective Expression; BP, Bodily Pain; DAS, Dyadic Adjustment Scale; DAS tot, DAS total scores; Dy Coh, Dyadic Cohesion; Dy Con, Dyadic Consensus; Dy Sat, Dyadic Satisfaction; ERF, Emotional Role Functioning; GHP, General Health Perceptions; MH, Mental Health; MSPSS, Multidimensional Scale of Perceived Social Support; PF, Physical Functioning; PRF, Physical Role Functioning; SCIM, Spinal Cord Independence Measure; SCIM, SCIM scores; SF, Social Functioning; SS SO, spousal support; SS Fam, family support; SS Fri, friends' support; SS Tot, MSPSS total scores; VT, Vitality.

Table 3 Correlations between the DAS and MSPSS scores

\begin{tabular}{|c|c|c|c|c|c|c|c|c|c|c|}
\hline & Dy Coh & Dy Sat & Aff Exp & Dy Con & DAS tot & SS SO & SS Fam & SS Fri & SS tot & SCIM \\
\hline Dy Coh & 1 & & & & & & & & & \\
\hline Dy Sat & $-0.35^{*}$ & 1 & & & & & & & & \\
\hline Aff Exp & $0.31^{*}$ & $0.36^{*}$ & 1 & & & & & & & \\
\hline Dy Con & 0.03 & $0.34^{*}$ & $0.69 * *$ & 1 & & & & & & \\
\hline DAS tot & $0.42 * *$ & $0.52 * *$ & $0.82^{* *}$ & $0.80 * *$ & 1 & & & & & \\
\hline SS SO & -0.04 & $0.54 * *$ & $0.54 * *$ & $0.61^{* *}$ & $0.63^{* *}$ & 1 & & & & \\
\hline SS Fam & 0.22 & 0.26 & $0.38^{*}$ & 0.27 & $0.43^{* *}$ & $0.34 *$ & 1 & & & \\
\hline SS Fri & 0.08 & 0.09 & 0.016 & 0.16 & 0.19 & 0.07 & 0.26 & 1 & & \\
\hline SS tot & 0.14 & $0.41^{* *}$ & $0.48^{* *}$ & $0.48^{* *}$ & $0.58^{* *}$ & $0.63^{* *}$ & $0.79 * *$ & $0.66^{* *}$ & 1 & \\
\hline SCIM & -0.00 & -0.07 & -0.18 & -0.04 & -0.08 & -0.08 & -0.02 & 0.12 & 0.02 & 1 \\
\hline
\end{tabular}

Abbreviations: Aff Exp, Affective Expression; Dy Coh, Dyadic Cohesion; Dy Con, Dyadic Consensus; Dy Sat, Dyadic Satisfaction; DAS, Dyadic Adjustment Scale; DAS tot, DAS total scores; MSPSS, Multidimensional Scale of Perceived Social Support; SCIM, Spinal Cord Independence Measure; SCIM, SCIM scores; SS Fam, family support; SS Fri, friends' support; SS SO, spousal support; SS tot, MSPSS total scores.

$* P<0.05$ (two-tailed).

$* * P<0.01$ (two-tailed).

Table 4 Correlations between the DAS and Short Form 36 scores

\begin{tabular}{|c|c|c|c|c|c|c|c|c|c|c|c|c|c|}
\hline Dy Coh & 1 & & & & & & & & & & & & \\
\hline Dy Sat & $-0.35^{*}$ & 1 & & & & & & & & & & & \\
\hline Dy Con & 0.03 & $0.34^{*}$ & $0.69 * *$ & 1 & & & & & & & & & \\
\hline DAS tot & $0.42^{* *}$ & $0.52^{* *}$ & $0.82^{* *}$ & $0.80^{* * *}$ & 1 & & & & & & & & \\
\hline PF & 0.02 & 0.22 & 0.10 & $0.24^{*}$ & 0.26 & 1 & & & & & & & \\
\hline GHP & 0.10 & -0.04 & -0.06 & -0.05 & 0.00 & 0.30 & $0.53^{* *}$ & $0.32^{*}$ & 1 & & & & \\
\hline VT & 0.07 & 0.29 & $0.38^{*}$ & 0.28 & $0.37^{*}$ & $0.41^{* *}$ & $0.31^{*}$ & 0.03 & 0.14 & 1 & & & \\
\hline SF & 0.08 & 0.14 & 0.21 & 0.16 & 0.22 & 0.21 & $0.43^{* *}$ & 0.02 & $0.38^{*}$ & $0.44^{* *}$ & 1 & & \\
\hline ERF & 0.23 & 0.04 & 0.10 & 0.05 & 0.18 & 0.10 & 0.28 & -0.02 & 0.28 & $0.45^{* *}$ & $0.40 * *$ & 1 & \\
\hline $\mathrm{MH}$ & 0.18 & $0.32 *$ & $0.40^{* *}$ & $0.37^{*}$ & $0.50^{* *}$ & 0.28 & 0.27 & 0.01 & $0.31^{*}$ & $0.66^{* *}$ & $0.48^{* *}$ & $0.59^{* *}$ & 1 \\
\hline
\end{tabular}

Abbreviations: Aff Exp, Affective Expression; BP, Bodily Pain; DAS, Dyadic Adjustment Scale; DAS tot, DAS total scores; Dy Coh, Dyadic Cohesion; Dy Con, Dyadic Consensus; Dy Sat, Dyadic Satisfaction; ERF, Emotional Role Functioning; GHP, General Health Perceptions; MH, Mental Health; PF, Physical Functioning; PRF, Physical Role Functioning; SF, Social Functioning; VT, Vitality. $* P<0.05$ (two-tailed)

${ }^{* *} P<0.01$ (two-tailed).

satisfaction itself seems not to be significantly conditioned by functional status. Moreover, couple and family relationships seem to be the most important sources of support when compared with informal social networks such as friendships. This finding is paralleled by the fact that friends' support is negatively correlated with age, suggesting a restriction in the social participation of older people. It is plausible that ageing persons might rely mostly on family support, and relevant psychological theories about motivation and emotional satisfaction across the life span suggest that the elderly might be more selective than younger people in choosing and regulating their involvement in close relationships. ${ }^{25}$

However, such a finding deserves special attention and further investigation with larger samples, as vast surveys on persons with 
physical limitations confirm that social support might weaken across the life cycle and that friends' support could be a significant buffer against depressive symptoms. ${ }^{26}$ Moreover, in our study population, friends' support is positively correlated with a higher satisfaction from physical role functioning, which refers to the impact of health on work or other activities. This finding suggests the presence of a significant association between effective support from social networks and social participation. Cross-cultural studies might also be of help in shedding light on the contribution of societal and cultural variables, as well as of social and environmental barriers.

With respect to spousal support, many correlations between such a support and measures of relationship quality were found. In more detail, spousal support is significantly related to the overall perception of relationship quality, as well as with specific domains such as dyadic satisfaction, affective expression and dyadic consensus. This infers a strong interdependence and a reciprocal influence between the perception of actual support and relationship quality. As confirmed by previous contributions, the perception of genuine support is more likely to occur within the context of satisfactory relationships, and, in turn, episodes of effective support can enhance couple satisfaction and reciprocal trust. ${ }^{27}$ Interestingly, the overall satisfaction from couple relationship and specific aspects such as affective expression are also correlated with family support, suggesting significant cross effects between different but interdependent relationships. Indeed, individuals are at the same time involved in multiple relationships in a complex set of interwoven systems and subsystems, and the mutual influence of couple and family relationships, as well as between the nuclear and extended family, is well established in research and theoretical perspectives on family relationships and families facing serious illnesses. $^{28}$

Relationship quality shows positive correlations also with different aspects of HRQoL, such as vitality and mental health, confirming the evidence of a relevant association between psychological well-being and satisfaction from intimate relationships. Interestingly, no correlation between HRQoL and spousal support was found. Such findings suggest that not only is social support strictly dependent on relationship quality but also that it might be less likely related to HRQoL than relationship quality. This is consistent with the hypothesis that social support cannot be separated from the relational context in which it takes place, and that the satisfaction from couple relationship could be more important for well-being than the perception of specific helping behaviours.

More to the point, behaviours have meanings, and supporting behaviours have special meanings in conditions in which the balance between togetherness and separateness, as well as between autonomy and interdependence, can be threatened by physical limitations. As previously said, disease conditions and physical limitations can have an impact on relationships with significant others, sometimes eliciting imbalances and skews in couple and family relationships. These include the heightening of closeness and interdependence, with the possible consequence of the perception of reduced autonomy and reciprocity. Such aspects are crucial in building healthy and satisfactory couple relationships, and also in defining frameworks for the interpretations of supporting behaviours. Providing or receiving support without losing active roles in significant relationships is indeed a key factor in couple and family adjustment to disease conditions or physical limitations.

Our data seem to confirm that, despite such potential problems, satisfactory couple relationships might be not so rare in SCI populations, and that physical impairment might be not so strictly related to marital satisfaction. In this respect, it must be remembered that the frequencies of the neurological categories and the functional status average score suggest that most of the people in our study population have acceptable levels of autonomy. A larger sample, with different clinical characteristics, might be of help for a more in-depth examination of the relationship between physical limitations and marital satisfaction.

This said, the disease evolution has to be taken into account as well, as SCI has typically a sudden impact and a relatively constant course, although medical complications can arise. As emphasised in relational models of adjustment to illness, ${ }^{28}$ in these kinds of conditions the crisis phase is the one that requires the most notable efforts in terms of adaptation, whereas in the long period of chronicity couples and families have time to adjust to the limitations imposed by the disease and to rebalance relationships after the crisis phase. This is usually more difficult in the case of progressive diseases, in which the condition worsens and poses on couples and families new and everchanging psychosocial demands.

The clinical implications of these data are worthy of attention as well, as they could contribute to inform treatment choices in psychological interventions. In fact, together with individual interventions, couple and family counselling or therapy could be fruitful options of treatment. Working on relationship quality, not simply in a psycho-educational vein, could be an important step to enhancing personal and relational well-being. As we have seen, social support cannot be seen as a sequence of isolated and discreet behaviours, as it happens within the context of meaningful relationships. Thus, improving communication skills in the context of couple relationships and, when necessary, providing couple therapy could be a useful approach for ameliorating relationships that can have a strong impact on QoL, social participation and even treatment adherence.

In conclusion, some study limitations should be also highlighted. In particular, the small sample size requires caution in the interpretation of the results, and it does not allow the creation of large enough subgroups for valid comparisons, such as those concerning gender differences or neurological categories, which could be extremely interesting in this context. Furthermore, the small sample size does not allow the use of more complex statistical tests, such as multivariate analysis, which could be helpful to examine more in-depth the relationships among the variables.

\section{DATA ARCHIVING}

There were no data to deposit.

\section{CONFLICT OF INTEREST}

The authors declare no conflict of interest.

1 Beedie A, Kennedy P. Quality of social support predicts hopelessness and depression post spinal cord injury. J Clin Psychol Med S 2002; 9: 227-234.

2 Elliott TR, Herrick SM, Patti AM, Witty TE, Godshall FJ, Spruell M. Assertiveness, social support, and psychological adjustment following spinal cord injury. Behav Res Ther 1991; 29: 485-493

3 Rintala D, Young ME, Hart KA, Clearman RR, Fuhrer MJ. Social support and the well-being of persons with spinal cord injury living in the community. Rehabil Psychol 1992; 37: 155-163.

4 Müller R, Peter C, Cieza A, Geyh S. The role of social support and social skills in people with spinal cord injury: A systematic review of the literature. Spinal Cord 2012; 50: 94-106.

5 Revenson TA, Schiaffino KM, Majerovitz SD, Gibofsky A. Social support as a doubleedged sword: The relation of positive and problematic support to depression among rheumatoid arthritis patients. Soc Sci Med 1991; 33: 807-813.

6 Fiske V, Coyne JC, Smith DA. Couples coping with myocardial infarction: An empirical reconsideration of the role of overprotectiveness. J Fam Psychol 1991; 5: 4-20. 
7 Kiecolt-Glaser JK, Newton TL. Marriage and health: His and hers. Psychol Bull 2001; 127: 472-503.

8 Coyne JC, DeLongis A. Going beyond social support: the role of social relationships in adaptation. J Consult Clin Psychol 1986; 54: 454-460.

9 Rook K. Detrimental aspects of social relationships: Taking stock of an emerging literature. In: Veil O, Baumann U (eds). The Meaning and Measurement of Social Support. Hemisphere: New York, pp 157-170, 1992.

10 Pierce GR, Sarason IG, Sarason BR. General and relationship-based perceptions of social support: Are two constructs better than one? J Pers Soc Psychol 1991; 61 1028-1039.

11 Holicky R, Charlifue S. Ageing with spinal cord injury: The impact of spousal support Disabil Rehabil 1999; 21: 250-257.

12 DeVivo MJ, Hawkins LN, Richards JS, Go BK. Outcomes of post-spinal cord injury marriages. Arch Phys Med Rehabil 1995; 76: 130-138.

13 Post MW, van Leeuwen CM. Psychosocial issues in spinal cord injury: A review. Spinal Cord 2012; 50: 382-389.

14 Alexander CJ, Hwang K, Sipski ML. Mothers with spinal cord injuries: Impact on marital, family, and children's adjustment. Arch Phys Med Rehabil 2002; 83: 24-30.

15 Chan RC, Lee PW, Lieh-Mak F. Coping with spinal cord injury: Personal and marital adjustment in the Hong Kong Chinese setting. Spinal Cord 2000; 38: 687-696.

16 Peters LC, Stambrook M, Moore AD, Zubek E, Dubo H, Blumenschein S. Differential effects of spinal cord injury and head injury on marital adjustment. Brain Inj 1992; 6: 461-467.

17 Yim SY, Lee IY, Yoon SH, Song MS, Rah EW, Moon HW. Quality of marital life in Korean spinal cord injured patients. Spinal Cord 1998; 36: 826-831.

18 Frazier PA, Tix AP, Barnett CL. The relational context of social support: Relationship satisfaction moderates the relations between enacted support and distress. Pers Soc Psychol Bull 2003; 29: 1133-1146.
19 Rolland JS. In sickness and in health: The impact of illness on couples' relationships. J Marital Fam Ther 1994; 20: 327-347.

20 Spanier GB. The measurement of marital quality. J Sex Marital Ther 1976; 5: 288-300.

21 Zimet GD, Powell SS, Farley GK, Werkman S, Berkoff KA. Psychometric characteristics of the Multidimensional Scale of Perceived Social Support. J Pers Assess 1990; 55: 610-617.

22 Ware JE, Sherbourne CD. The MOS 36-Item Short-Form Health Survey (SF-36): I. conceptual framework and item selection. Med Care 1992; 30: 473-483.

23 Itzkovich M, Gelernter I, Biering-Sorensen F, Weeks C, Laramee MT, Craven BC et al. The Spinal Cord Independence Measure (SCIM) version III: reliability and validity in a multi-center international study. Disabil Rehabil 2007; 29: 1926-1933.

24 Gentili P, Contreras L, Cassaniti M, D’Arista F. La Dyadic Adjustment Scale. Una misura dell'adattamento di coppia. Minerva Psichiatr 2002; 43: 107-116.

25 Carstensen LL, Isaacowitz DM, Charles ST. Taking time seriously: A theory of socioemotional selectivity. Am Psychol 1999; 54: 165-181.

26 Jensen MP, Smith AE, Bombardier CH, Yorkston KM, Mirò J, Molton IR. Social support, depression, and physical disability: age and diagnostic group effects. Disabil Health $\mathrm{J}$ 2014; 7: 164-172.

27 Cutrona CE, Russell DW, Gardner KA. The relationship enhancement model of social support. In: Revenson TA, Kayser K, Bodenmann G (eds). Couples Coping with Stress. Emerging Perspectives on Dyadic Coping. American Psychological Association: Washington, DC, pp 73-95.

28 Rolland JS. Families, IIIness and Disability. An Integrative Treatment Model. Basic Books: New York, 1994.

29 DeVivo MJ, Biering-Sørensen F, New P, Chen Y. International Spinal Cord Injury Data Set. Standardization of data analysis and reporting of results from the International Spinal Cord Injury Core Data Set. Spinal Cord 2011; 49: 596-599. 